Bull. Mater. Sci., Vol. 36, No. 1, February 2013, pp. 9-14. (c) Indian Academy of Sciences.

\title{
X-ray reflectivity study of bias graded diamond like carbon film synthesized by ECR plasma
}

\author{
R M DEY ${ }^{\dagger, *}$, S K DESHPANDE ${ }^{\ddagger}$,S B SINGH ${ }^{\#}$, N CHAND ${ }^{\#}$, D S PATIL ${ }^{\#}$ and S K KULKARNI \\ DST Unit on Nanoscience, Department of Physics, University of Pune, Pune 411 007, India \\ $\dagger$ Present Address: CTSR, Department of Materials Science and Engineering, SUNY Stony Brook, Stony Brook, \\ NY 11794, USA \\ †UGC-DAE Consortium for Scientific Research, " Laser and Plasma Technology Division, Bhabha Atomic Research \\ Centre, Mumbai 400 085, India
}

MS received 21 June 2009; revised 15 July 2012

\begin{abstract}
Diamond like carbon (DLC) coatings were deposited on silicon substrates by microwave electron cyclotron resonance (ECR) plasma CVD process using plasma of $\mathrm{Ar}$ and $\mathrm{CH}_{4}$ gases under the influence of negative d.c. self bias generated on the substrates by application of RF (13.56 MHz) power. The negative bias voltage was varied from $-60 \mathrm{~V}$ to $-150 \mathrm{~V}$ during deposition of DLC films on Si substrate. Detailed X-ray reflectivity (XRR) study was carried out to find out film properties like surface roughness, thickness and density of the films as a function of variation of negative bias voltage. The study shows that the DLC films constituted of composite layer i.e. the upper sub surface layer followed by denser bottom layer representing the bulk of the film. The upper layer is relatively thinner as compared to the bottom layer. The XRR study was an attempt to substantiate the sub-plantation model for DLC film growth.
\end{abstract}

Keywords. Diamond like carbon; electron cyclotron resonance; X-ray reflectivity; roughness; sub-plantation model; amorphous material.

\section{Introduction}

Carbon is a unique element in terms of formation of compounds with vast majority of elements of periodic table. The allotropic forms of carbon (Robertson 2002) are diamond $\left(100 \% s p^{3}\right)$, graphite $\left(100 \% s p^{2}\right)$ and buckyball. It exists in amorphous form and hydrogenated diamond like carbon is one of the amorphous forms of carbon. In hydrogenated diamond like carbon (H-DLC), carbon has both hybridization $s p^{3}$ and $s p^{2}$, and sometimes $s p$. Amorphous DLC possesses a very high potential as a technological material. Due to its unique combination of properties like diamond and the ease with which it can be synthesized, DLC has found its vast application in electronics, medical, space, defense, nuclear, fields etc. Though the extent of properties shown by DLC films are inferior to those shown by diamond films, they have advantages in terms of deposition at room temperature, deposition onto polymer, plastic, iron substrates with very smooth surface finish and are cheaper to produce than diamond. DLC is mainly grown by the deposition of hyperthermal species onto various substrates. The property of DLC films depends on the deposition technique and the operating conditions. A vast majority of techniques like filtered cathodic

\footnotetext{
*Author for correspondence (ravi.dey@ sunysb.edu)
}

vacuum arc deposition, laser ablation, direct ion beam, magnetron sputtering, microwave, electron cyclotron resonance (ECR) microwave techniques have been used for the deposition of DLC on various substrates (Lifshitz 1996; Dey et al 2008). Many applications like optical, biomedical, electronics demand extremely smooth surface and exact knowledge about surface topography. Further the thickness determination is crucial in many applications in electronics, thin film capacitors, optics, etc. The X-ray reflectivity (XRR) technique has great applicability in these applications. It is a nondestructive technique for determining layer thickness, surface and interfacial roughness and internal structure of films. The XRR technique is alike the optical reflectivity technique and is performed at a glancing angle.

To understand and predict properties of DLC films and carbon system in general, it is necessary to have an idea about the growth mechanism of the disordered film which confers the typical properties characteristics of the films. Different theoretical growth models for understanding the nucleation and growth mechanism were proposed for DLC films like sub-plantation model (Davis 1993; Robertson 1994; Uhlmann et al 1998), atomic peening model (Koponen et al 1995), pressure induced compressive stress model (McKenzie et al 1991) and cylindrical spike model (Hofsäss et al 1998). Marks (2005) has discussed different growth mechanisms of DLC. Further to resolve the 
theoretical issues concerned with the growth mechanism, computational studies were also carried out by molecular dynamics assuming different potentials like Tersoff potential (Tersoff 1988), Brenner potential (Brenner 1990), Environment dependent interaction potential (EDIP) (Marks 2001, 2005), many-body potential (Kaukonen and Nieminen 1992; Kelires 1992, 1993; Marks et al 1996), ab initio MD technique utilizing Car Parrinello code, density-functional based tight-binding (DFTB) method (Lifshitz et al 1990; Uhlmann et al 1998) and Monte Carlo simulations for DLC growth using Trydin code (Lifshitz et al 1990). All these studies were carried out to gain knowledge about the mechanism for promotion of $s p^{3}$ bonding of carbon.

Recently, experimental studies were carried out on the DLC films grown by hyperthermal carbon species (Lifshitz et al 2007; Dey et al 2008). Lifshitz et al (2007) had shown by AFM studies that the films deposited by low energetic carbon species were rough whereas the films deposited with higher energy were smoother. In the bias graded deposited DLC films by electron cyclotron resonance plasma, Dey et al (2008) conducted a series of experiments like XPS, AFM, spectroscopic ellipsometry, contact angle measurement and found that for the films deposited at low bias voltage the films had low $s p^{3} / s p^{2}$ ratio, contact angle, refractive index and high roughness in comparison with the films deposited at high bias voltage. In all the experimental studies, the results were explained on the basis of sub-plantation model. However, in the studies direct evidence regarding the prediction of the sub-plantation model was not given like presence of layering with different densities and thickness. Hence, it was realized to conduct experiment for checking the validity of sub-plantation model and thereby substantiate it. XRR technique was chosen due to its unique probing technique and capabilities in the estimation of film properties like density, thickness, roughness and internal layering. Further with the help of XRR studies the role of hyperthermal species and applicability of the models for other systems different from carbon system can be explored.

\section{Experimental}

For XRR studies, thin film of DLC were deposited on silicon substrates by microwave ECR plasma CVD process. The experimental deposition consisted of a water cooled magnetron source giving microwave radiation at 1.5 kilo-watts power and frequency of $2.45 \mathrm{GHz}$. The description of the system is discussed elsewhere (Dey et al 2008).

Prior to deposition of DLC, the silicon substrates were ultrasonically cleaned in isopropyl alcohol for $\sim 20 \mathrm{~min}$. The substrates were loaded in the system and the system was pumped down to a base pressure of $\sim 1.5 \times 10^{-6}$ mbar. The substrates were further sputtered by applying negative bias of $-100 \mathrm{~V}$ by RF power of $13.6 \mathrm{MHz}$. The operating parameters are described in table 1.

For XRR study, DLC films were loaded on a Seifert X-ray diffractometer 2003 XRD 230 TT. $\mathrm{Cu} \mathrm{K}_{\alpha}$ with a wavelength
Table 1. Operating parameters for DLC deposition on Si substrate by microwave ECR plasma.

$\begin{array}{ll}\text { Base pressure } & \sim 1.5 \times 10^{-6} \mathrm{mbar} \\ \text { Carrier gas } & \text { Argon }(1-2 \mathrm{SCCM}) \\ \text { Operating pressure for Ar plasma } & \sim 2.5 \times 10^{-4} \mathrm{mbar} \\ \text { Microwave power } & \sim 300 \text { watts } \\ \text { Precursor gas } & \text { Methane }(1-2 \mathrm{SCCM}) \\ \text { Final deposition pressure } & \sim 5.5 \times 10^{-4} \mathrm{mbar} \\ \text { Deposition time } & \sim 15-20 \text { minutes } \\ \text { Applied bias voltage } & -60 \mathrm{~V} \text { to }-150 \mathrm{~V}\end{array}$

$1.542 \AA$ was used as X-ray source. For proper aligning of the sample and calibration, a rocking scan for each sample was carried out before reflectivity measurement. For XRR, $2 \theta$ was taken up to $5^{\circ}$ with a step size of $0 \cdot 001^{\circ}$.

\section{Results and discussion}

The films deposited were hard and could not be scratched by sharp stainless steel probe. The XRR patterns show fringes due to interference effect. From the spacing of the fringes a qualitative estimation about the film thickness can be estimated. Figure 1 shows XRR pattern for DLC films deposited from $-60 \mathrm{~V}$ to $-150 \mathrm{~V}$. It can be seen that the film with $-60 \mathrm{~V}$ and $-150 \mathrm{~V}$ have few fringes as compared to the films deposited at $-100 \mathrm{~V}$. The fringe spacing and thickness are related to each other by the equation (Spiller 1994; Kondrashov et al 1997)

$$
\begin{aligned}
& m \lambda=2 t \sqrt{\alpha_{m}^{2}-\alpha_{c}^{2}}, \\
& \Rightarrow t=\frac{m \lambda}{2 \sqrt{\alpha_{m}^{2}-\alpha_{c}^{2}}} .
\end{aligned}
$$

In terms of $m$ and $(m+1)_{\text {th }}$ fringe, the thickness $t$ is given as

$$
\Rightarrow t=\frac{\lambda / 2}{\sqrt{\alpha_{m+1}^{2}-\alpha_{c}^{2}}-\sqrt{\alpha_{m}^{2}-\alpha_{c}^{2}}},
$$

where $\lambda$ is wavelength of X-ray used, $\alpha_{\mathrm{c}}$ the critical angle, the maximum angle for total reflection for X-ray, $\alpha_{m}$ the angle corresponding to the $m$ th fringe and $\alpha_{m+1}$ the angle corresponding to $(m+1)$ th fringe.

From (3) we see that $t$ is large with the films with large number of fringes within a unit $\alpha$ range and vice versa. Hence, by observing the patterns it seems that DLC films deposited at $-60 \mathrm{~V}$ and $-150 \mathrm{~V}$ are thinner than the films deposited at $-100 \mathrm{~V}$ and $-150 \mathrm{~V}$.

From mere observation of the pattern it is very difficult to describe the internal layering of the films. Hence to solve this problem, fitting of the XRR pattern was carried out by Parratt32 software. Parratt32 is based on Parratt's (Nielsen 

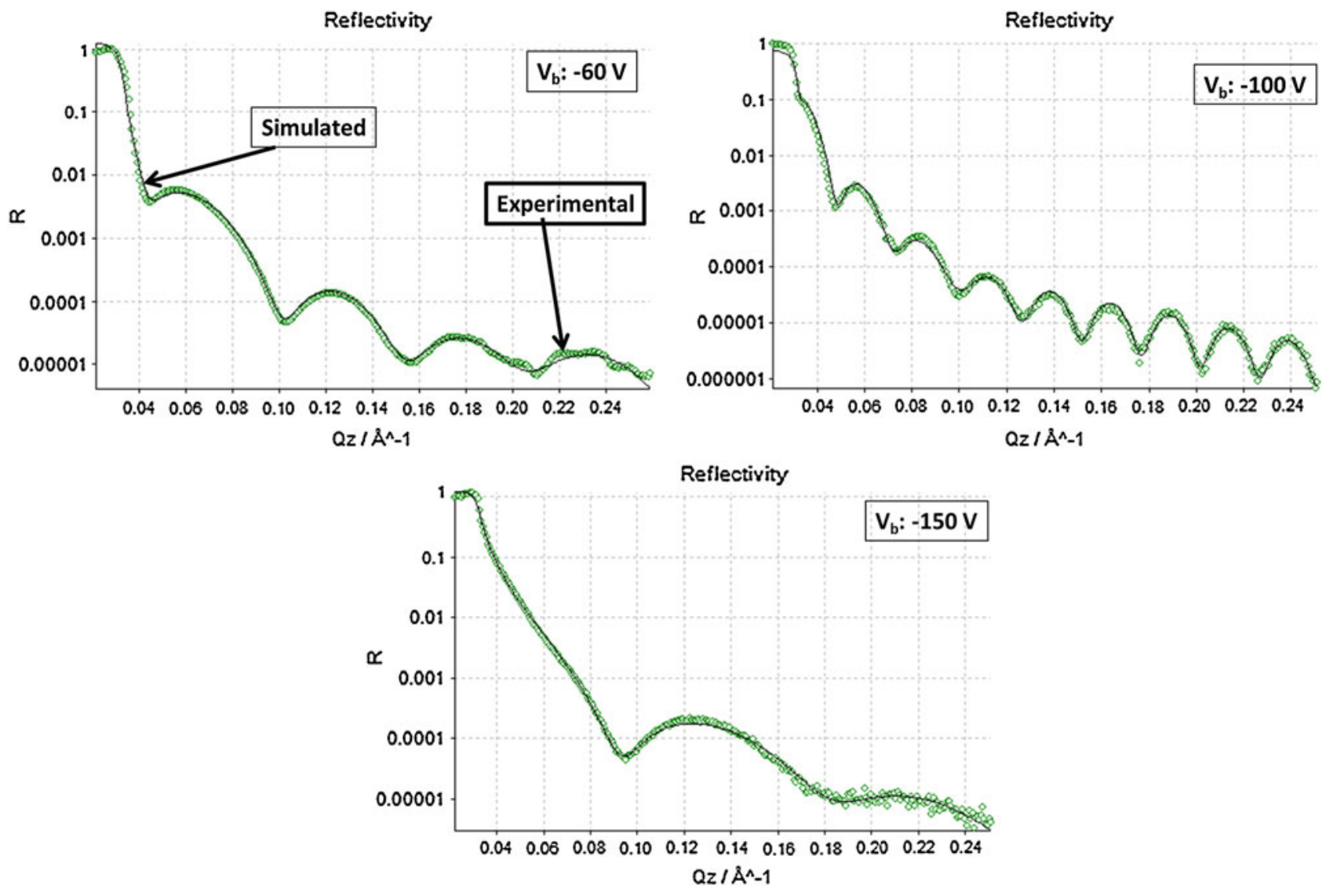

Figure 1. Fitting of XRR curve by Parrat32 reflectivity tool for DLC films biased at (a) $-60 \mathrm{~V},(\mathbf{b})-100 \mathrm{~V}$ and (c) $-150 \mathrm{~V}$.

and McMorrow 2001) recursive formula using Fresnel's equations for the calculation of reflectivity from a film with $N$ layers being used as given

$$
r_{N-1, N}=\frac{r_{N-1, N}^{\prime}+r_{N, \infty}^{\prime} p_{N}^{2}}{1+r_{N-1, N}^{\prime} r_{N, \infty}^{\prime} p_{N}^{2}},
$$

where $p_{N}^{2}=e^{i t_{N} Q_{N}}$ is the phase factor, $t_{N}$ the thickness of the $N$ th layer, $Q_{N}=2 k_{N} \sin \alpha_{N}, k_{N}$ the wave vector and $\alpha_{N}$ the angle of incidence on $N$ th layer.

Using (4), the total reflectivity from interface between vacuum or air and first layer is procured. Finally an algorithm is used to minimize the difference in the absolute intensity between the experimental curve and the simulated curve as the parameters (density, roughness, thickness, absorption etc.) are adjusted by the computer.

For fitting, four layers were tried for each film and as seen from figure 1 the simulated and experimental curves almost coincided. From this fitting the final values for thickness, $t$, of each layer, roughness, $\sigma$, and density were obtained. In table 2, the summary of the fitted parameters is shown. The interpretation of different layers added in the XRR fitting can be understood from figure 2 .

Figure 3 shows variation of surface roughness with applied bias voltage. It can be seen that the roughness for the films deposited at $-60 \mathrm{~V}(7.5 \AA)$ and $-150 \mathrm{~V}(7 \AA)$ is higher
Table 2. Summary of XRR parameter obtained from simulation.

\begin{tabular}{lccc}
\hline Sample & $-60 \mathrm{~V}$ & $-100 \mathrm{~V}$ & $-150 \mathrm{~V}$ \\
\hline Roughness $(\AA)$ & & & \\
$\quad$ SSL & 8 & 2 & 7 \\
SSTL & 5 & 10 & 12 \\
BL & 4 & 10 & 11 \\
IL & 2 & 13 & 7 \\
Si & 6 & 4 & 6 \\
Sarratt & & & \\
SSL & $7 \cdot 8 \mathrm{E}-6$ & $8 \cdot 70 \mathrm{E}-6$ & $6 \cdot 715 \mathrm{E}-6$ \\
SSTL & $1 \cdot 077 \mathrm{E}-5$ & $1 \cdot 404 \mathrm{E}-5$ & $9 \cdot 759 \mathrm{E}-6$ \\
BL & $1.471 \mathrm{E}-5$ & $1 \cdot 538 \mathrm{E}-5$ & $1 \cdot 504 \mathrm{E}-5$ \\
IL & $2 \cdot 45 \mathrm{E}-5$ & $2 \cdot 47 \mathrm{E}-5$ & $2 \cdot 207 \mathrm{E}-5$ \\
Si & $2 \cdot 098 \mathrm{E}-5$ & $2 \cdot 161 \mathrm{E}-5$ & $2 \cdot 037 \mathrm{E}-5$ \\
Thickness $(\AA)$ & & & \\
SSL & 32 & 14 & 11 \\
SSTL & 17 & 6 & 10 \\
BL & 55 & 225 & 44 \\
IL & 5 & 8 & 9
\end{tabular}

IL: Interlayer between DLC film and substrate, BL: Bulk layer of DLC, SSTL: Sub-surface transition layer and SSL: Sub-surface layer.

as compared to the films deposited at $-100 \mathrm{~V}(2 \AA)$. As seen from figure 4 , the thickness of DLC films deposited 


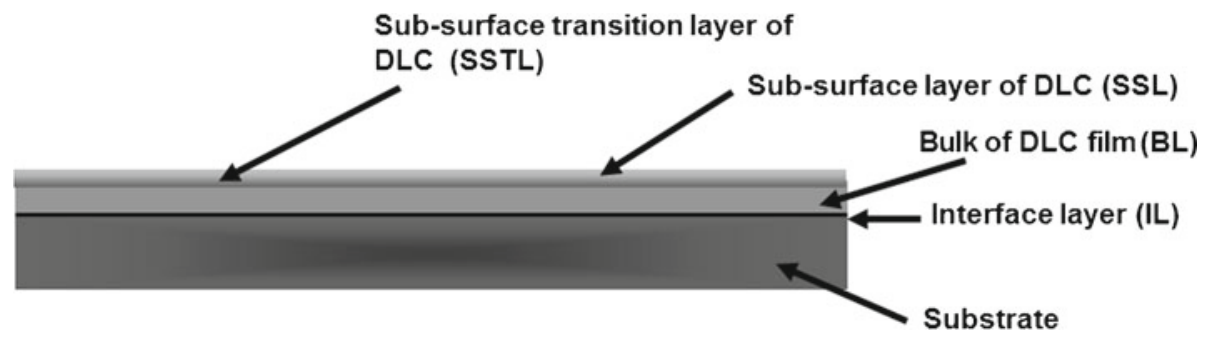

Figure 2. Schematic of four layers fitting in XRR.

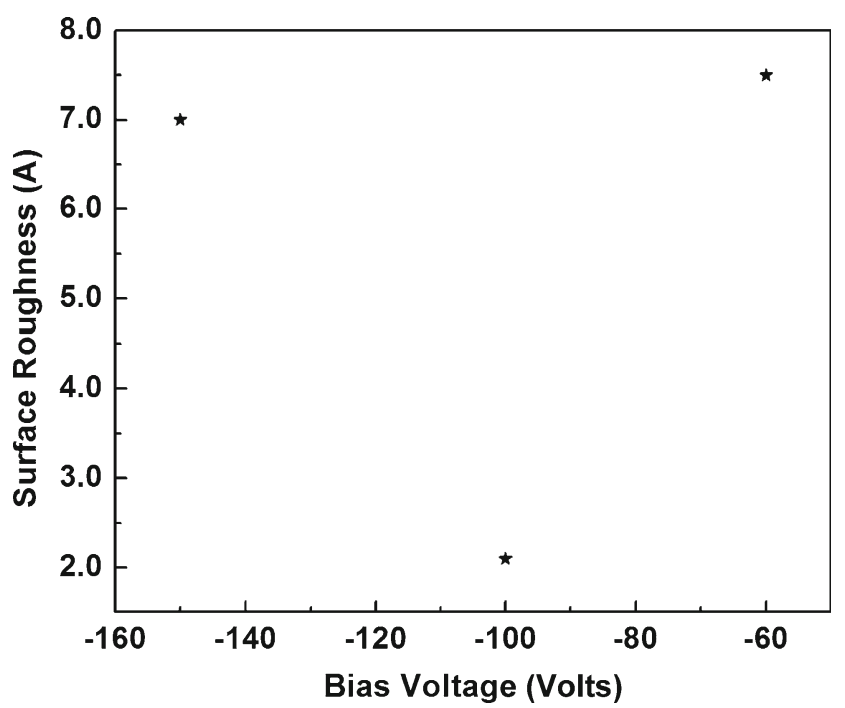

Figure 3. Surface roughness variation of DLC films with bias voltage.

at $-100 \mathrm{~V}$ is higher as compared to the films deposited at $-60 \mathrm{~V}$ and $-150 \mathrm{~V}$. Also it is evident from the graph that the thickness of the subsurface of DLC film and interface of the film and silicon is smaller than the bulk of the film. A similar trend is observed for the variation of density of the subsurface and bulk of DLC films with bias voltage as shown in figure 5. It is further seen that there is difference in density between the two layers of DLC films. The subsurface is of lower density as compared to the bulk of DLC film. To allow a smooth transition a sub transition layer is included in the model whose density is intermediate to that of bulk of DLC film and sub surface part of the film. The density of the films deposited at $-60 \mathrm{~V}$ and $-150 \mathrm{~V}$ is lower as compared to the films deposited at $-100 \mathrm{~V}$. The highest density obtained is $1.87 \mathrm{~g} / \mathrm{cc}$ for the film deposited at $-100 \mathrm{~V}$.

Thus it can be clearly observed from the results that the XRR study carried out on DLC films indicates the film to be composed of two layers i.e. the upper subsurface layer of lower density and thickness as compared to the lower layer constituting the bulk of the film.

The density obtained by fitting the experimental curve is shown in figure 5. It can be observed that the density of the interfacial layer is highest compared to the other layers and varies within the range of $2 \cdot 7-3 \cdot 1 \mathrm{~g} / \mathrm{cm}^{3}$. This value is intermediate between the densities of pure $\operatorname{Si}\left(2.329 \mathrm{~g} / \mathrm{cm}^{3}\right)$ and $\mathrm{SiC}\left(3 \cdot 21 \mathrm{~g} / \mathrm{cm}^{3}\right)$. The density value indicates formation of $\mathrm{SiC}$ bonding in the interfacial layer when the carbon ions are incorporated in the film. Hence we can conclude that the film is well adhered to the Si substrate. The lower density value for interfacial layer compared to pure $\mathrm{SiC}$ can be understood from the deposition mechanism of the DLC coating. During deposition of the coating along with carbon radical, hydrogen also gets incorporated in the substrate. Hence due to this the density decreases compared to $\mathrm{SiC}$.

The present XRR data can be readily interpreted in terms of the sub-plantation model. For low energy of the carbon species, $E_{i}<E_{\text {critical }}$, the surface mobility of the carbon species on account of it is low activation energy, which is large as compared to the surface penetration and forms island like structure of high $s p^{2}$ content leading to appreciable surface roughness of the films (Lifshitz et al 1990; Peng et al 2001; Dey et al 2008). Consequently, the density of the film is low as can be seen for DLC film deposited at $-60 \mathrm{~V}$. When the energy of carbon species is equal to the critical energy $\left(E_{\text {critical }}\right)$, the hyperthermal species have sufficient energy to penetrate the surface and get trapped in the interstitial sites in the sub-surface, a few layers below the surface and thus leading to the sub-plantation phenomenon. The continuous entrapment leads to high densification of the films as is evident from DLC films grown at $-100 \mathrm{~V}$. In this narrow energy window the film grows internally rather than surface diffusion process leading to smoother film. As the impinging carbon species energy, $E_{i}$, becomes significantly higher than $E_{\text {critical }}$, energy collisional damage throughout the bulk of the film is pronounced resulting in more roughness and less densification of the films as is seen in the case of $-150 \mathrm{~V}$ deposited DLC film. Due to the collisional damage the growth of film is also hindered and thickness of the film is less as compared to the films deposited for same period and at $E_{\text {critical }}$. Molecular dynamic simulation result of Uhlmann et al (1998) predicts that DLC film is composed of two composite layers, the upper sub-surface rich in $s p^{2}$ bonded carbon with more voids and the lower part with more $s p^{3}$ bonded carbon content and denser than top layer. As the film grows, the successive layers are compressed and the carbon species get preferentially converted to $s p^{3}$ leaving the sub-surface rich in $s p^{2}$ bonded carbon content and less dense than the underlying bulk layer. From 

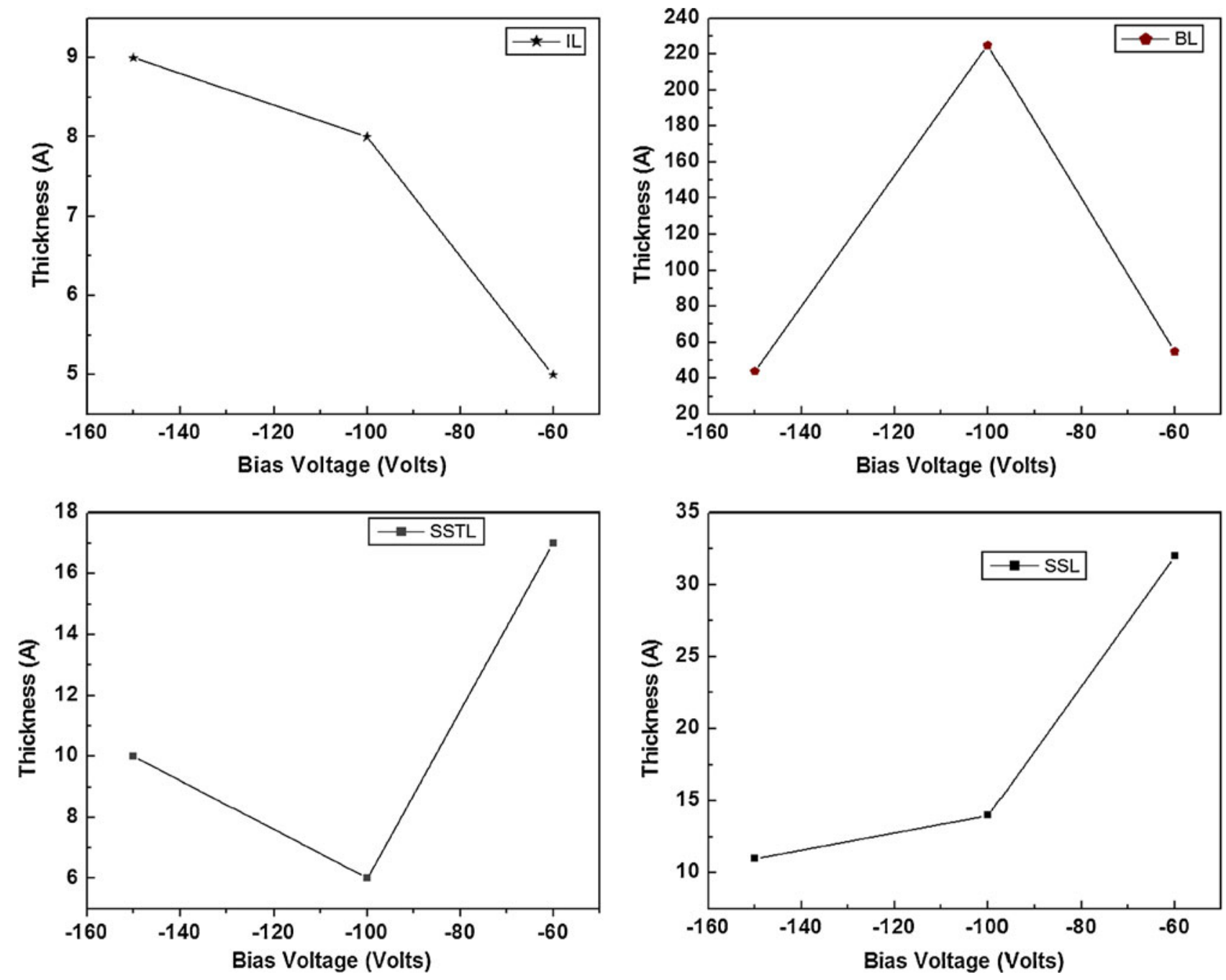

Figure 4. Thickness of different layers of DLC films grown at different bias voltages. (IL: Interlayer between DLC film and substrate. BL: bulk layer of DLC. SSTL: sub-surface transition layer and SSL: sub-surface layer).

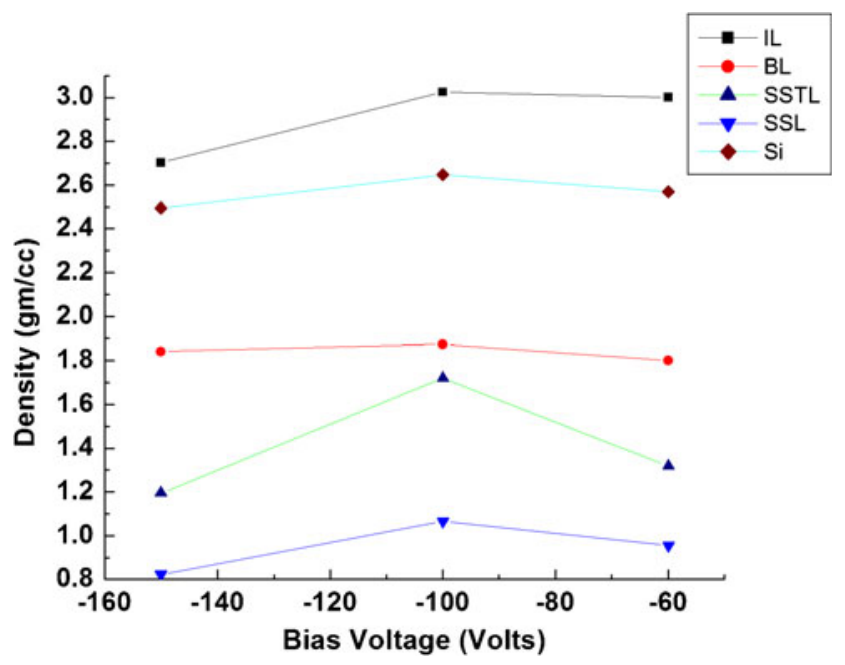

Figure 5. Density variation of top and bottom layers of DLC films with bias voltage. (IL: Interlayer between DLC film and substrate. BL: bulk layer of DLC. SSTL: sub-surface transition layer and SSL: sub-surface layer). figure 4 it is seen that the XRR study clearly supports the simulated result for the DLC growth under various impinging energies. The results of XRR studies are in accordance with the experimental results that at around $100 \mathrm{~V}$ the properties of DLC are optimized and are more diamond like (Robertson 2008).

\section{Conclusions}

The detailed XRR studies conducted on the bias graded deposited DLC films substantiates the simulated result of subplantation model. The XRR studies clearly supports the composite layer structure for DLC film with the bottom layer denser as compared to the top layer. It further supports the density variation of the films as a function of the impinging energy of the carbon species. The XRR studies further reveal the correlation between surface roughness of the films and bias voltage. For lower energy the film is rough and for critical incident energy window the film attains minimum roughness. Thereafter, for higher energy the roughness of the 
film increases. The findings are in accordance with the subplantation model and clearly supports the simulated results of the model.

\section{Acknowledgements}

The authors thank UGC-DAE Consortium for Scientific Research, for the X-ray diffraction facility. (RMD) and (SBS) would like to acknowledge the financial support given by the Department of Atomic Energy, Government of India, by awarding research fellowships to them during the course of the work reported here and (SKK) thanks UGC, India, for continuous support.

\section{References}

Brenner D W 1990 Phys. Rev. Lett. 429458

Davis C A 1993 Thin Solid Films 22630

Dey R M et al 2008 Curr. Appl. Phys. 86

Hofsäss H, Feldermann H, Merk R, Sebastian M and Ronning C 1998 Appl. Phys. A66 153

Kaukonen H-P and Nieminen R M 1992 Phys. Rev. Lett. 68620

Kelires P C 1992 Phys. Rev. Lett. 681855

Kelires P C 1993 Phys. Rev. B47 1829
Kondrashov P E, Smirnov I S, Novoselova E G, Yu Yablokov S and Baranov A M 1997 Diam. Relat. Mater. 61784

Koponen I, Hakovirta M and Lappalainen R 1995 J. Appl. Phys. 78 5837

Lifshitz Y 1996 Diam. Relat. Mater. 5388

Lifshitz Y, Kasi S R, Rabalais J W and Eckstein W 1990 Phys. Rev. B41 10468

Lifshitz Y, Edrei R, Hoffman A, Grossman E, Lempert G D, Berthold J, Schultrich B and Jäger H U 2007 Diam. Relat. Mater. 161771

Marks N A 2001 Phys. Rev. B63 035401

Marks N A 2005 Diam. Relat. Mater. 141223

Marks N A, McKenzie D R and Pailthorpe B A 1996 Phys. Rev. B53 4117

McKenzie D R, Muller D and Pailthorpe B A 1991 Phys. Rev. Lett. 67773

Nielsen J A and McMorrow D 2001 Elements of modern X-ray physics (England: Wiley)

Peng X L, Barber Z H and Clyne T W 2001 Surf. Coat. Technol. 13823

Robertson J 1994 Diam. Relat. Mater. 3361

Robertson J 2002 Mater Sci. Eng. R37 129

Robertson J 2008 in Tribology of diamond like carbon films (eds) Christopher Donnet and Ali Erdemier (Springer) p. 15

Spiller E 1994 Soft X-ray optics (Washington: SPIE Optical Engineering Press)

Tersoff J 1988 Phys. Rev. Lett. 612879

Uhlmann S, Frauenheim Th and Lifshitz Y 1998 Phys. Rev. Lett. 81 641 